\title{
Excitation of a uniformly moving atom through vacuum fluctuations
}

\author{
Anatoly A. Svidzinsky (1) \\ Department of Physics \& Astronomy, Texas A\&M University, College Station, Texas 77843, USA
}

(Received 7 August 2019; published 16 October 2019)

\begin{abstract}
In systems with broken Lorentz invariance the vacuum state of the field can depend on the choice of the inertial reference frame. This leads to observable effects. For example, the motion of an atom with a constant velocity above a flat metal surface can yield atomic excitation with simultaneous emission of a surface plasmon, while uniform motion of the atom through an optical cavity can yield atomic excitation with emission of a photon into cavity modes. We calculate the probability of these processes and make a connection with the Cherenkov radiation of a neutral atom uniformly moving through a medium and Unruh radiation of the atom accelerated in free space. In all these cases the moving atom becomes excited and the field quantum is emitted through vacuum fluctuations at the expense of the kinetic energy of the atom.
\end{abstract}

DOI: 10.1103/PhysRevResearch.1.033027

\section{INTRODUCTION}

In free space, the quantum vacuum is Lorentz invariant, so a uniformly moving observer would not see any effect due to motion, but an accelerated observer would. For example, an atom accelerated through Minkowski vacuum can become excited. This is known as the Unruh effect [1] (or FullingDavies-Unruh effect in full [1-3]). Interpretation of the effect depends on the choice of the reference frame.

A noninertial observer having a proper constant acceleration $a$, i.e., a Rindler observer [4], sees that space is filled with thermal photons with Unruh temperature $T_{U}$ proportional to the acceleration [1]

$$
T_{U}=\frac{\hbar a}{2 \pi k_{B} c} .
$$

From the perspective of the accelerated observer the groundstate atoms accelerated through the Minkowski vacuum will be promoted to an excited state by absorption of the Rindler particles (Unruh effect) [1]. However, an inertial observer interprets the absorption of a Rindler particle as the emission of a Minkowski particle [5], which is known as acceleration radiation. A similar mechanism yields excitation of an atom freely falling in gravitational field [6] or a fixed atom in Minkowski space in the presence of an accelerated mirror [7].

It is necessary to reach accelerations on the order of $\sim 10^{20} \mathrm{~m} / \mathrm{s}^{2}$ to obtain an Unruh radiation corresponding to a temperature of a few kelvins. That is effect is very small. As a consequence, direct experimental evidence for the Unruh effect is still lacking. One closely related observation in laboratories is the electron depolarization in storage rings [8], which can be connected to the "circular Unruh effect" [9-12].

Published by the American Physical Society under the terms of the Creative Commons Attribution 4.0 International license. Further distribution of this work must maintain attribution to the author(s) and the published article's title, journal citation, and DOI.
Strong enhancement of radiation can be produced by a fast nonadiabatic switch of the interaction of atoms with the field at the boundaries of a cavity [13].

Dependence of the field vacuum state on a choice of noninertial reference frame leads to the acceleration radiation. In one frame the field could be in a vacuum state, but in another frame the same state of the field can contain particles which can excite the atom.

In systems with broken Lorentz invariance the vacuum state can also depend on a choice of the inertial reference frame. The presence of matter breaks Lorentz invariance of the quantum vacuum. As a consequence, an atom uniformly moving relative to matter can become excited by a mechanism similar to that of the Unruh effect. Cherenkov radiation produced by a uniformly moving atom through a medium is an example. It can be understood based on the energy and momentum conservation during photon emission $[14,15]$.

For a nonrelativistic motion of the atom with velocity $\mathbf{V}$ through a medium with the refractive index $n$ the conservation equations in the laboratory frame read

$$
\begin{gathered}
\hbar \mathbf{k}+\Delta \mathbf{p}=0 \\
\mathbf{V} \cdot \Delta \mathbf{p}+\Delta E_{\text {atom }}+\hbar v=0
\end{gathered}
$$

where $\hbar \mathbf{k}$ and $\hbar v=\hbar c k / n$ are the momentum and energy of the emitted photon, $\Delta \mathbf{p}$ is the change of the atom's momentum, $\mathbf{V} \cdot \Delta \mathbf{p}$ is the change of the atom's kinetic energy, and $\Delta E_{\text {atom }}$ is the change of the atom's internal energy.

Equations (2) and (3) give

$$
\Delta E_{\text {atom }}=\hbar k V\left(\cos \theta-\frac{c}{V n}\right),
$$

where $\theta$ is the photon emission angle relative to $\mathbf{V}$. Equation (4) shows that if the photon is emitted inside the Cherenkov cone $(\cos \theta>c / V n)$ then $\Delta E_{\text {atom }}>0$; that is, the atom becomes excited [14-16]. This is analogous to the Unruh acceleration radiation for which an inertial observer sees that the accelerated atom becomes excited by emitting a photon. 
In both cases the energy is gained from the atom's kinetic energy. If emission occurs outside the Cherenkov cone then $\Delta E_{\text {atom }}<0$ and the atom must go from the excited to the ground state.

In the reference frame moving with velocity $\mathbf{V}$ the photon frequency shifts and becomes

$$
v^{\prime}=\frac{\frac{c k}{n}-\mathbf{V} \cdot \mathbf{k}}{\sqrt{1-\frac{V^{2}}{c^{2}}}} .
$$

If $V>c / n$ there are photons with negative frequency. The wave frequency becomes negative if it is measured in a coordinate system which is moving faster than the phase velocity of the wave. A change in the sign of the frequency of the wave between two inertial reference frames corresponds to a reversal of the phase velocity. Yet from the point of view of the relation $E=\hbar \nu$, a positive quantum of energy apparently becomes a negative-energy one [17].

The behavior of the negative-energy quanta is essential to a kinematics of amplification of waves which can explain certain amplification phenomena [18]: a variety of travelingwave-tube-type amplifiers [19], a resistive-wall amplifier [20], and amplification of ultrasound waves in semiconductors [21]. The negative-energy quanta also play a role in Cherenkov radiation, quantum friction, and the Hawking radiation. The quantum friction is predicted to occur between relatively moving dielectrics at zero temperature [22,23] and stems from the mixing of positive- and negative-frequency waves in the two materials [24,25]. Quantum friction can also occur in rotating dielectric bodies [26-28]. At zero temperature, vacuum friction transforms mechanical energy into light emission and heats the rotating body. Hawking radiation [29] and its laboratory analogs $[30,31]$ originate from the change in the sign of the frequency of a wave as it crosses the event horizon.

In the uniformly moving frame the field Hamiltonian consistent with the canonical commutation relations $\left[\hat{b}_{v}, \hat{b}_{v}^{+}\right]=1$ reads $[16,32]$

$$
\hat{H}=\sum_{v^{\prime}} \hbar v^{\prime} \hat{b}_{v^{\prime}}^{+} \hat{b}_{v^{\prime}}
$$

where $\hat{b}_{v^{\prime}}^{+}$is the creation operator for a photon in the medium with the Doppler-shifted frequency $v^{\prime}$. Since in the frame moving with $V>c / n$ there are photons with negative frequencies, in this frame there is no lower bound on the photon energy.

From the perspective of the moving observer, photons emitted inside the Cherenkov cone have negative energy [33] which excites the atom, while photons emitted outside the Cherenkov cone have positive energy which deexcites the atom.

Next we investigate a similar problem with translational symmetry along the $y, z$, and $t$ axes which somewhat resembles Cherenkov radiation in the medium and Unruh acceleration radiation of an atom moving through the Minkowski vacuum.

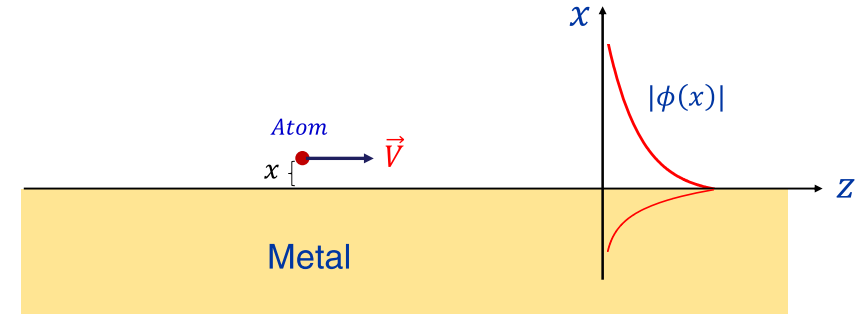

FIG. 1. Atom is moving with constant velocity $V$ above metal surface and becomes excited by emitting a surface plasmon. Electromagnetic field of the surface plasmon exponentially decays away from the metal surface.

\section{EXCITATION OF AN ATOM UNIFORMLY MOVING ABOVE A METAL SURFACE}

Here we consider a flat interface between a metal and vacuum (see Fig. 1). In such system there exist collective excitations of the electromagnetic field and metal electrons which propagate along the surface, known as surface plasmons [34]. Electromagnetic field in the surface plasmon exponentially decays away from the surface as shown in Fig. 1. For example, in the Drude model of metal if we omit dissipation the frequency-dependent dielectric function reads

$$
\varepsilon(v)=1-\frac{\omega_{p}^{2}}{v^{2}},
$$

and the field outside the metal is given by [34]

$$
\phi_{\nu}(t, \mathbf{r})=e^{-i v t+i k z-\alpha x},
$$

where $v$ is the mode frequency which is related to the plasmon wave number $k$ through the dispersion relation

$$
v^{2}=\frac{\omega_{p}^{2}}{2}+c^{2} k^{2}-\sqrt{\frac{\omega_{p}^{4}}{4}+c^{4} k^{4}},
$$

$\omega_{p}=\sqrt{\frac{n_{e} e^{2}}{\varepsilon_{0} m_{e}}}$ is the electron plasma frequency, $n_{e}$ is the density of free electrons of mass $m_{e}$, and $\alpha$ is the inverse of the decay length which depends on $k$,

$$
\alpha^{2}=\sqrt{\frac{\omega_{p}^{4}}{4 c^{4}}+k^{4}}-\frac{\omega_{p}^{2}}{2 c^{2}} .
$$

We assume that in the laboratory frame the plasmon field is in a vacuum state.

In the inertial frame moving with velocity $\mathbf{V}$ along the metal surface the mode frequency is Doppler shifted and becomes

$$
v^{\prime}=\frac{v-k V}{\sqrt{1-\frac{V^{2}}{c^{2}}}} .
$$

In the moving frame the plasmon frequency is negative for modes with $k>\sqrt{\frac{c^{2}-V^{2}}{2 c^{2}-V^{2}}} \omega_{p} / V$. When phase velocity of the wave is smaller than medium velocity the energy of the quantum which propagates opposite to the medium motion is negative. Negative frequency implies that the vacuum state in the moving frame is different from the vacuum in the laboratory frame, which yields observable effects. For 
example, a ground-state atom moving above the metal surface with a constant velocity can become excited. A classical analogy of this effect has been discussed in Ref. [35], while the rate of spontaneous emission by a moving two-level atom interacting with the near field of a plasmonic slab has been calculated in Ref. [36]. Optical instabilities in moving media are linked to a spontaneous parity-time-symmetry breaking of the system [37].

Interpretation of the effect depends on the choice of the inertial reference frame. In the laboratory frame the atom becomes excited by emitting a surface plasmon with positive energy at the expense of kinetic energy of the atom. In the moving frame the atom becomes excited by emitting a surface plasmon with negative energy which insures energy conservation.

Next we calculate the excitation probability. We will perform calculations in the laboratory frame. In this frame the atom's trajectory is

$$
t=\frac{\tau}{\sqrt{1-\frac{V^{2}}{c^{2}}}}, \quad z=\frac{V \tau}{\sqrt{1-\frac{V^{2}}{c^{2}}}},
$$

where $\tau$ is the proper time of the atom. The interaction Hamiltonian between the atom moving at a distance $x$ above the metal and the surface plasmon with frequency $v$ reads

$$
\hat{H}_{\text {int }}(\tau)=\hbar g\left\{\hat{a}_{v} \phi_{\nu}[t(\tau), \mathbf{r}(\tau)]+\text { H.c. }\right\}\left(\hat{\sigma} e^{-i \omega_{a} \tau}+\text { H.c. }\right),
$$

where $\hat{a}_{v}$ is the plasmon annihilation operator, $\hat{\sigma}$ is the atomic lowering operator, $\omega_{a}$ is the atomic frequency in the atom's frame, and $g$ is the atom-plasmon coupling constant. Since the atom feels the local value of the field, the field mode function in Eq. (12) is taken at the atom's location $t(\tau), x, z(\tau)$.

Excitation of the atom with simultaneous emission of a plasmon is due to the counterrotating term $\hat{a}_{v}^{+} \hat{\sigma}^{+}$in the interaction Hamiltonian. The probability $P$ that the atom with transition frequency $\omega_{a}$ becomes excited and a plasmon with frequency $v$ is emitted is given by the integral from the matrix element between the initial state of the system in which the atom is in the ground state $|b\rangle$ and there are no plasmons and the final state in which the atom is in the excited state $|a\rangle$ and one surface plasmon with frequency $v$ is present:

$$
\begin{aligned}
P & =\frac{1}{\hbar^{2}}\left|\int_{0}^{t} d \tau\left\langle 1_{\nu}, a\left|\hat{H}_{\text {int }}(\tau)\right| 0, b\right\rangle\right|^{2} \\
& =g^{2} e^{-2 \alpha x}\left|\int_{0}^{t} d \tau e^{i v t(\tau)-i k z(\tau)} e^{i \omega_{a} \tau}\right|^{2} .
\end{aligned}
$$

Inserting here Eq. (11) we obtain

$$
P=g^{2} e^{-2 \alpha x}\left|\int_{0}^{t} d \tau e^{i\left(v^{\prime}+\omega_{a}\right) \tau}\right|^{2},
$$

where $v^{\prime}$ is the plasmon frequency in the reference frame of the moving atom.

The mode for which $v^{\prime}=-\omega_{a}$ is in resonance. For this mode the integral in Eq. (14) linearly grows with time. If $\omega_{a} \gg \omega_{p}$ and $V \ll c$ then for the resonant mode $k=\omega_{a} / V$. The motion of the atom yields emission of the surface plasmon into this resonant mode with simultaneous excitation of the atom with probability

$$
P=g^{2} e^{-2 \alpha x} t^{2}
$$

where $t$ is the time of the atom's motion above the metal surface.

In order to increase excitation probability, instead of atoms, one can shoot polar molecules above the metal surface. The rotational excitations of molecules are in the microwave regime and have an anharmonic energy spectrum. The anharmonicity allows us to pick out a two-level subspace in the rotational spectrum and treat the molecular ensemble as a twolevel system. Trapping molecules close to the metal surface yields a strong electric dipole coupling with $g \sim 10^{4} \mathrm{~Hz}$ [38].

For $\omega_{a}=10^{10} \mathrm{~Hz}$ and velocity $V=10^{3} \mathrm{~m} / \mathrm{s}$ the field decay length outside the metal for the resonant mode is $V / \omega_{a}=$ $100 \mathrm{~nm}$. So, in order to achieve strong coupling, one should shoot the molecules closer than $100 \mathrm{~nm}$ to the surface. For these parameters the molecule will be excited with almost unit probability after moving $10 \mathrm{~cm}$ above the metal. Thus, the effect is not very small.

One should mention that the molecule experiences the Casimir-Polder interaction with the metal surface [39]. This interaction has the same origin as the Lamb shift of energy levels due to the zero-point fluctuations of the electromagnetic field. The presence of the surface alters the spectrum of the vacuum fluctuations. As a consequence, the molecule's energy shift depends on the distance to the surface, which results in an interaction potential. The Casimir-Polder force acting on the molecule is perpendicular to the flat surface. It decays as $1 / x^{4}$ for short distances $x$ and as $1 / x^{5}$ for large separation due to retardation.

For the molecule moving parallel to the metal surface the vacuum fluctuations can yield molecule excitation with simultaneous emission of the surface plasmon. This occurs at the expense of the molecule's kinetic energy which yields the appearance of a friction force opposite to the direction of the motion. Contrary to the Casimir-Polder interaction, the friction force decays exponentially with the distance to the surface $x$.

\section{EXCITATION OF OPTICAL VORTICES IN A CAVITY BY A MOVING ATOM}

Next we investigate a system with no translational symmetry. Namely, we consider a cylindrically symmetric cavity or cavity with a rectangular symmetry for which normal modes of the field are Laguerre-Gaussian (optical vortices) or Hermite-Gaussian, respectively. The Laguerre-Gaussian and Hermite-Gaussian modes form a complete set. The presence of the cavity breaks the Lorentz invariance of the field equations because the boundary conditions at the cavity mirrors depend on the reference frame.

We assume that the cavity is fixed in Minkowski space-time and a two-level ( $a$ and $b$ ) atom is moving through the cavity with a constant velocity $V$ along a straight line so that the atomic trajectory is given by the equations

$$
x=V t, \quad y=d, \quad z=0,
$$

where $d$ is the impact parameter and $z$ is the cavity axis (see Fig. 2). The atom is moving from infinity and passes through 


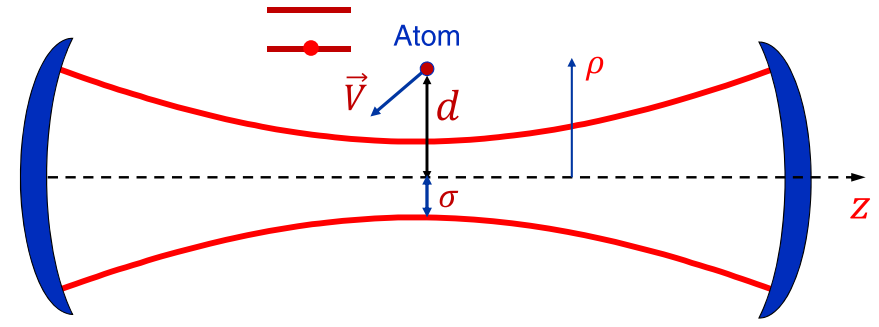

FIG. 2. A ground-state atom is moving through a cavity with a constant velocity at impact parameter $d$. Virtual transitions yield excitation of the atom and emission of a photon in cavity modes.

the center of the cavity in the direction perpendicular to the cavity axis. This effectively causes a change of the coupling between the atom and the cavity modes with time.

We assume that initially the atom is in the ground state $|b\rangle$ and there are no photons in the cavity modes. In the interaction picture the interaction Hamiltonian between the atom and a photon with frequency $v$ is given by Eq. (12) in which $\hat{a}_{v}$ is the photon annihilation operator, $\tau=t \sqrt{1-V^{2} / c^{2}}$ is the proper time for the atom, and $g$ is the coupling constant between the atom and the cavity mode $\phi_{v}(t, \mathbf{r})$. Since the atom feels the local value of the field, $\phi_{v}$ is taken at the atom's location $t(\tau), \mathbf{r}(\tau)$.

The change of the coupling between field and the atom with time can result in atomic excitation with simultaneous photon emission into the cavity modes due to the counterrotating terms $\hat{a}_{v}^{+} \hat{\sigma}^{+}$in the interaction Hamiltonian. The probability $P$ that the atom with transition frequency $\omega_{a}$ becomes excited and a photon in the mode $\phi_{v}(t, \mathbf{r})$ is generated is given by the integral

$$
P=g^{2}\left|\int_{-\infty}^{\infty} d \tau \phi_{\nu}^{*}[t(\tau), \mathbf{r}(t(\tau))] e^{i \omega_{a} \tau}\right|^{2} .
$$

Changing the integration variable to $t$ we obtain

$$
P=\left(1-\frac{V^{2}}{c^{2}}\right) g^{2}\left|\int_{-\infty}^{\infty} d t \phi_{v}^{*}[t, \mathbf{r}(t)] e^{i \omega t}\right|^{2},
$$

where $\omega=\sqrt{1-V^{2} / c^{2}} \omega_{a}$. Next we discuss excitation of the optical vortex cavity modes by the uniformly moving atom.

We assume that the cavity can support the LaguerreGaussian modes. For a cavity with a cylindrical symmetry, the Laguerre-Gaussian modes of frequency $v$ can be written as a function of the polar radius $\rho=\sqrt{x^{2}+y^{2}}$ and the azimuthal angle $\varphi$. We consider a subset of the Laguerre-Gaussian cavity modes in the form of optical vortices

$$
\begin{aligned}
\phi_{\nu}(t, \mathbf{r})= & \frac{e^{i m \varphi}}{w(z)} \frac{\rho^{|m|}}{w^{|m|}(z)} e^{-\rho^{2} / w^{2}(z)} \\
& \times \cos \left[\frac{v \rho^{2}}{2 c R(z)}-(|m|+1) \arctan \left(\frac{z}{z_{R}}\right)\right] e^{-i v t},
\end{aligned}
$$

where $m=0, \pm 1, \pm 2, \ldots$ is the azimuthal parameter, $z_{R}$ is the Rayleigh length, $w(z)$ and $R(z)$ are the beam radius and the radius of curvature,

$$
w(z)=\sigma \sqrt{1+\left(\frac{z}{z_{R}}\right)^{2}}, \quad R(z)=z\left[1+\left(\frac{z_{R}}{z}\right)^{2}\right],
$$

while $\sigma$ is the beam radius at the waist. The optical vortex carries orbital angular momentum $m \hbar$ per photon. We assume that the normalization factor in $\phi_{v}$ is subsumed under the coupling constant $g$.

For the modes (18) at the atomic position the mode function is

$$
\phi_{\nu}(t, \mathbf{r}(t))=\frac{[d+i \operatorname{sgn}(m) V t]^{|m|}}{\sigma^{|m|+1}} e^{-\left(V^{2} t^{2}+d^{2}\right) / \sigma^{2}} e^{-i v t},
$$

and Eq. (17) yields

$$
\begin{aligned}
P_{m}= & \left(1-\frac{V^{2}}{c^{2}}\right) \frac{g^{2}}{\sigma^{2}|m|+2} e^{-2 d^{2} / \sigma^{2}} \\
& \times\left|\int_{-\infty}^{\infty} d t[d-i \operatorname{sgn}(m) V t]^{|m|} e^{-V^{2} t^{2} / \sigma^{2}+i(\omega+v) t}\right|^{2} .
\end{aligned}
$$

For $m=0$ the integration over time reduces to the following integral:

$$
\int_{-\infty}^{\infty} d t e^{-\alpha^{2} t^{2}+i b t}=\frac{\sqrt{\pi}}{|\alpha|} e^{-b^{2} / 4 \alpha^{2}} .
$$

To find the time integral for arbitrary $m$, namely,

$$
\int_{-\infty}^{\infty} d t e^{-\alpha^{2} t^{2}+i b t}(d-i \alpha t)^{|m|}
$$

we rewrite Eq. (21) as

$$
\int_{-\infty}^{\infty} d t e^{-\alpha^{2} t^{2}+i b t+\gamma(d-i \alpha t)}=\frac{\sqrt{\pi}}{|\alpha|} e^{-b^{2} / 4 \alpha^{2}} e^{-\gamma^{2} / 4+\gamma(d+b / 2 \alpha)} .
$$

Differentiating over $\gamma$ from both sides $|m|$ times we obtain

$$
\begin{aligned}
& \int_{-\infty}^{\infty} d t e^{-\alpha^{2} t^{2}+i b t}(d-i \alpha t)^{|m|} \\
& \quad=\left.\frac{\sqrt{\pi}}{|\alpha|} e^{-b^{2} / 4 \alpha^{2}} \frac{d^{|m|}}{d \gamma^{|m|}} e^{-\gamma^{2} / 4+\gamma(d+b / 2 \alpha)}\right|_{\gamma=0} .
\end{aligned}
$$

Taking into account that Hermite polynomials $H_{m}(x)$ are given by the exponential generating function, namely,

$$
e^{-\gamma^{2} / 4+x \gamma}=\sum_{m=0}^{\infty} H_{m}(x) \frac{\gamma^{m}}{2^{m} m !},
$$

we obtain

$$
\left.\frac{d^{|m|}}{d \gamma^{|m|}} e^{-\gamma^{2} / 4+x \gamma}\right|_{\gamma=0}=\frac{H_{|m|}(x)}{2^{|m|}} .
$$

As a result, the integral over time is given by

$$
\begin{aligned}
& \int_{-\infty}^{\infty} d t e^{-\alpha^{2} t^{2}+i b t}(d-i \alpha t)^{|m|} \\
& =\frac{\sqrt{\pi}}{2^{|m|}|\alpha|} e^{-b^{2} / 4 \alpha^{2}} H_{|m|}\left(d+\frac{b}{2 \alpha}\right) .
\end{aligned}
$$




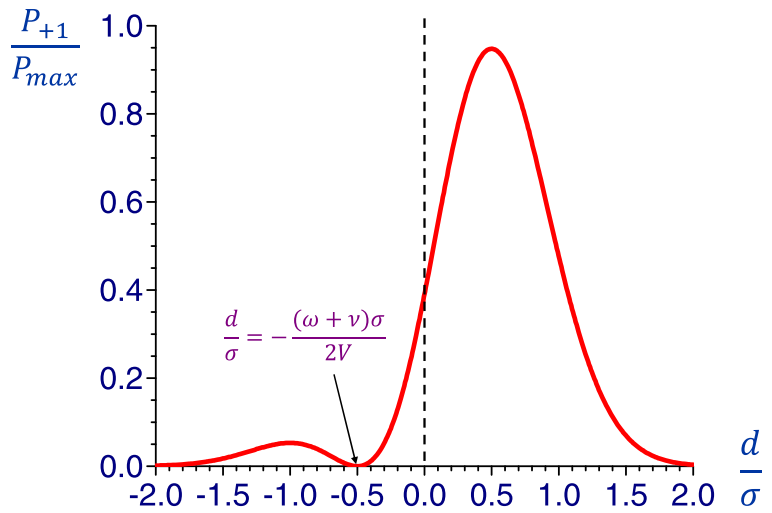

FIG. 3. Excitation probability of an optical vortex with the right vorticity $m=+1$ in a cavity by a uniformly moving atom as a function of the impact parameter $d$ for $\sigma(\omega+v) / 2 V=0.5$. For $d>0(d<0)$ the atom moves in the same (opposite) direction as the energy flow in the vortex. The probability is normalized by its maximum value.

This yields the following answer for the probability that the atom becomes excited and a photon in the mode $m$ is emitted:

$$
\begin{aligned}
P_{m}= & \left(1-\frac{V^{2}}{c^{2}}\right) \frac{\pi g^{2}}{2^{2|m|} V^{2}} H_{|m|}^{2}\left(\frac{d}{\sigma}+\operatorname{sgn}(m) \frac{\sigma(\omega+v)}{2 V}\right) \\
& \times \exp \left(-\frac{2 d^{2}}{\sigma^{2}}-\frac{(\omega+v)^{2} \sigma^{2}}{2 V^{2}}\right) .
\end{aligned}
$$

Equation (22) shows that modes with opposite direction of the orbital angular momentum are excited with different probability. For example, for $m= \pm 1$ the Hermite polynomial reduces to $H_{1}(u)=2 u$ and

$$
\begin{aligned}
P_{ \pm 1}= & \left(1-\frac{V^{2}}{c^{2}}\right) \frac{\pi g^{2}}{V^{2}}\left(\frac{d}{\sigma} \pm \frac{\sigma(\omega+v)}{2 V}\right)^{2} \\
& \times \exp \left(-\frac{2 d^{2}}{\sigma^{2}}-\frac{(\omega+v)^{2} \sigma^{2}}{2 V^{2}}\right) .
\end{aligned}
$$

If $d V>0$ the optical vortex with the right vorticity is excited with greater probability. Moreover, if $d V=\sigma^{2}(\omega+$ $v) / 2$ the left vorticity mode $m=-1$ is not becoming excited at all.

Figure 3 shows the probability of excitation of an optical vortex with the right vorticity $P_{+1}$ as a function of the impact parameter $d$ for $\sigma(\omega+v) / 2 V=0.5$. For $d>0$ the atom moves in the same direction as the energy flow in the vortex and excitation probability is larger. This is intuitive from the perspective of the angular momentum conservation. Namely, angular momentum of the translational atomic motion is transferred into the orbital angular momentum of the field.

Nevertheless, even for $d<0$ when the atom moves in the direction opposite to the energy flow in the vortex the excitation probability of the vortex mode is nonzero. This, however, does not contradict the conservation of the angular momentum because vortex modes are excited in pairs with positive and negative $m$ and the average orbital angular momentum of the generated vortex pair is in the direction of the angular momentum of the atom's motion. For example, the average angular momentum of the $m= \pm 1$ vortex pair is

$$
\begin{aligned}
\hbar P_{+1}-\hbar P_{-1}= & 2\left(1-\frac{V^{2}}{c^{2}}\right) \frac{\pi \hbar g^{2}(\omega+v) d}{V^{3}} \\
& \times \exp \left(-\frac{2 d^{2}}{\sigma^{2}}-\frac{(\omega+v)^{2} \sigma^{2}}{2 V^{2}}\right) .
\end{aligned}
$$

This expression is positive for $d>0$ and negative otherwise.

The excitation probability (22) is governed by an exponential factor

$$
\exp \left(-\frac{2 d^{2}}{\sigma^{2}}-\frac{(\omega+v)^{2} \sigma^{2}}{2 V^{2}}\right)
$$

which is usually small. To avoid exponential suppression of excitation one should satisfy conditions

$$
d \lesssim \sigma, \quad V \gtrsim(\omega+v) \sigma .
$$

The latter condition can be approximately written as

$$
\frac{V}{c} \gtrsim 2 \pi \frac{\sigma}{\lambda}
$$

where $\lambda=2 \pi c /(\omega+v)$ is the wavelength corresponding to the net excitation energy of the atom and the field $\hbar(\omega+\nu)$. The excitation probability is largest if $V \sim c$ and $\sigma \sim \lambda$. For such parameters

$$
P_{\max } \sim \frac{\wp_{a b}^{2} v^{2}}{\hbar c^{3}} \sim 0.001
$$

where $\wp_{a b}$ is the atomic dipole moment matrix element.

Ground-state atoms emit photons into the cavity modes due to the counterrotating terms $\hat{a}_{v}^{+} \hat{\sigma}^{+}$in the Hamiltonian and absorb photons already present in the cavity by means of the usual resonant absorption described by the terms $\hat{a}_{v} \hat{\sigma}^{+}$. The probability of photon absorption $P^{\text {abs }}$ is given by Eq. (16) in which $\phi_{v}^{*}$ is replaced with $\phi_{v}$. This is equivalent to change $v \rightarrow-v, m \rightarrow-m$ in Eqs. (22) which yields the following relation between probability of absorption and emission:

$$
P^{\mathrm{abs}}=\frac{H_{|m|}^{2}\left(\frac{d}{\sigma}-\operatorname{sgn}(m) \frac{\sigma(\omega-v)}{2 V}\right)}{H_{|m|}^{2}\left(\frac{d}{\sigma}+\operatorname{sgn}(m) \frac{\sigma(\omega+v)}{2 V}\right)} \exp \left(\frac{2 \omega \nu \sigma^{2}}{V^{2}}\right) P^{\mathrm{exc}} .
$$

If the atomic beam is injected into the cavity then after a sufficiently long time the cavity field will reach a steady state. Statistics of photons in the cavity modes can be obtained using the quantum master equation technique, as developed in the quantum theory of the laser [40]. If atoms are ejected randomly the equation of motion for the density matrix of the cavity field mode is [13]

$$
\begin{aligned}
\frac{d \rho_{n, n}}{d t}= & -\Gamma_{e}\left[(n+1) \rho_{n, n}-n \rho_{n-1, n-1}\right] \\
& -\Gamma_{a}\left[n \rho_{n, n}-(n+1) \rho_{n+1, n+1}\right],
\end{aligned}
$$

where $\Gamma_{e}$ and $\Gamma_{a}$ are emission and absorption rates of the photon in the cavity. The ratio $\Gamma_{e} / \Gamma_{a}$ is equal to $P^{\text {exc }} / P^{\text {abs }}$. The steady-state solution of Eq. (25) exists when absorption is greater than emission $\left(P^{\mathrm{abs}}>P^{\mathrm{exc}}\right)$ and is given by thermal distribution

$$
\rho_{n, n}=\frac{\bar{n}_{v}^{n}}{\left(\bar{n}_{v}+1\right)^{n+1}},
$$




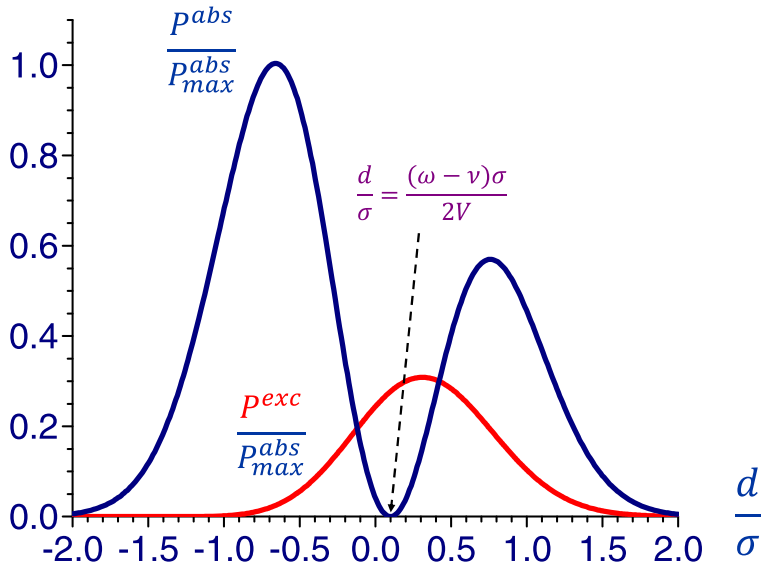

FIG. 4. Probability of emission and absorption into the vortex mode with $m=+1$ as a function of the impact parameter $d$ for $\sigma(\omega+v) / V=2.6$ and $\sigma(\omega-v) / V=0.2$. The plots are normalized to the maximum value of the probability of absorption $P_{\max }^{\text {abs }}$.

where the average photon number is

$$
\bar{n}_{v}=\frac{1}{e^{\frac{h v-\mu}{k_{B} T}}-1} .
$$

In this formula

$$
T=\frac{\hbar V^{2}}{2 k_{B} \omega \sigma^{2}}
$$

is the effective temperature which is independent of the impact parameter $d$, and $\mu$ is the effective chemical potential. In particular, for the modes with $m= \pm 1$

$$
\mu=\frac{\hbar V^{2}}{2 \omega \sigma^{2}} \ln \left[\frac{2 V d \pm \sigma^{2}(v+\omega)}{2 V d \pm \sigma^{2}(v-\omega)}\right] .
$$

One can vary the effective chemical potential at fixed $T$ by changing the impact parameter $d$. Here $\mu=\hbar v$ is a point of phase transition. At this point $P^{\mathrm{exc}}=P^{\mathrm{abs}}$. If $P^{\mathrm{exc}}>P^{\mathrm{abs}}$ there is no steady-state solution of Eq. (25) and field in the cavity grows with time indefinitely if we disregard cavity loses.

In Fig. 4 we plot probability of emission and absorption into the vortex mode with $m=+1$ as a function of the impact parameter $d$ for $\sigma(\omega+v) / V=2.6$ and $\sigma(\omega-v) / V=0.2$. For $d=(\omega-v) \sigma^{2} / 2 V$ the probability of absorption into the cavity mode vanishes. As a result, in the vicinity of this point $P^{\mathrm{exc}}>P^{\mathrm{abs}}$ and the field in the cavity grows with time.

\section{EXCITATION OF HERMITE-GAUSSIAN MODES IN A CAVITY}

Analogous calculations can be done for Hermite-Gaussian modes that are relevant to the rectangular geometry of the cavity and have field mode functions

$$
\begin{aligned}
\phi_{\nu}(t, \mathbf{r})= & \frac{1}{w(z)} H_{m}\left(\frac{\sqrt{2} x}{w(z)}\right) H_{n}\left(\frac{\sqrt{2} y}{w(z)}\right) e^{-\rho^{2} / w^{2}(z)} \\
& \times \cos \left[\frac{v \rho^{2}}{2 c R(z)}-(m+n+1) \arctan \left(\frac{z}{z_{R}}\right)\right] e^{-i v t},
\end{aligned}
$$

where $m, n=0,1,2, \ldots$
For the Hermite-Gaussian mode (30) the field mode function at the atomic position is

$$
\phi_{\nu}(t, \mathbf{r}(t))=\frac{e^{-i v t}}{\sigma} H_{m}\left(\frac{\sqrt{2} V t}{w_{0}}\right) H_{n}\left(\frac{\sqrt{2} d}{w_{0}}\right) e^{-\left(V^{2} t^{2}+d^{2}\right) / \sigma^{2}}
$$

and Eq. (17) yields for the probability of atom excitation with photon emission in the Hermite-Gaussian mode

$$
\begin{aligned}
P_{m, n}= & \left(1-\frac{V^{2}}{c^{2}}\right) \frac{g^{2}}{\sigma^{2}} e^{-2 d^{2} / \sigma^{2}} H_{n}^{2}\left(\frac{\sqrt{2} d}{\sigma}\right) \\
& \times\left|\int_{-\infty}^{\infty} d t H_{m}\left(\frac{\sqrt{2} V t}{\sigma}\right) e^{-V^{2} t^{2} / \sigma^{2}+i(\omega+v) t}\right|^{2} .
\end{aligned}
$$

Time integration can be calculated using the formula

$$
\begin{aligned}
\int_{-\infty}^{\infty} d t H_{m}(c t) e^{-\alpha^{2} t^{2}+i b t}= & \frac{(-1)^{m} \sqrt{\pi}\left(\alpha^{2}-c^{2}\right)^{m / 2}}{\alpha^{m+1}} e^{-b^{2} / 4 \alpha^{2}} \\
& \times H_{m}\left(\frac{b c}{2 \alpha \sqrt{c^{2}-\alpha^{2}}}\right),
\end{aligned}
$$

which gives

$$
\begin{aligned}
P_{m, n}= & \left(1-\frac{V^{2}}{c^{2}}\right) \frac{\pi g^{2}}{V^{2}} H_{n}^{2}\left(\frac{\sqrt{2} d}{\sigma}\right) H_{m}^{2}\left(\frac{(\omega+v) \sigma}{\sqrt{2} V}\right) \\
& \times \exp \left(-\frac{2 d^{2}}{\sigma^{2}}-\frac{(\omega+v)^{2} \sigma^{2}}{2 V^{2}}\right) .
\end{aligned}
$$

The probability of excitation is governed by a similar exponential factor as in the case of Laguerre-Gaussian modes.

\section{SUMMARY}

Virtual transitions governed by the counterrotating terms in the interaction Hamiltonian yield observable effects; e.g., they shift frequency of atomic or nuclear transitions (the Lamb shift). Here we discuss another manifestation of the virtual transitions, namely, excitation of a uniformly moving atom with simultaneous emission of the field quantum. We consider an atom moving with a constant velocity above a metal surface or through an optical cavity, calculate the excitation probability, and show that for realistic parameters the effect can be large enough to be observed experimentally. Instead of a single atom one can use atomic ensembles which could enhance excitation by orders of magnitude due to the collective (superradiant) contribution.

Interpretation of the effect depends on a choice of the reference frame. In the laboratory frame the moving atom becomes excited and the field quantum is emitted at the expense of the kinetic energy of the atom. Excitation of the uniformly moving atom through the optical cavity with simultaneous emission of a photon into the cavity can be understood as a sum combination resonance. Such resonance occurs when system parameters are modulated with the frequency equal to the sum of two normal-mode frequencies. Taking into account that the mode function of the photon in the cavity has the form

$$
\phi_{\nu}(t, \mathbf{r})=\phi_{v}(\mathbf{r}) e^{-i v t},
$$


the counterrotating term in the interaction Hamiltonian can be written as

$$
\hbar g \phi_{v}^{*}[\mathbf{r}(t)] e^{i(v+\omega) t} \hat{a}_{v}^{+} \hat{\sigma}^{+},
$$

where $\mathbf{r}(t)$ is the trajectory of the atom. One can interpret the factor $g \phi_{v}^{*}[\mathbf{r}(t)]$ as an effective coupling constant which depends on time due to atomic motion relative to the cavity. The Fourier component of $g \phi_{v}^{*}[\mathbf{r}(t)]$ proportional to $e^{-i(v+\omega) t}$ is in the sum combination resonance with the atom-field system. This resonance yields simultaneous excitation of the atom and the field mode from the vacuum.

The effect is somewhat similar to the Unruh acceleration radiation. A neutral atom accelerated in Minkowski vacuum in free space can become excited by emitting a photon (from the perspective of an inertial observer). In this case the effective coupling between light and the atom changes with time because of acceleration.

In the frame moving with the atom the kinetic energy of the atom is equal to zero. Thus, from the perspective of a moving observer the atom cannot become excited at the expense of its kinetic energy and the energy conservation must be satisfied by other means. For example, in the reference frame of an atom moving above the metal surface the energy of surface plasmons is negative for large enough wave vectors. From the perspective of the moving observer the atom becomes excited by emitting a resonant surface plasmon with negative energy which insures energy conservation. This example demonstrates that in systems with broken Lorentz invariance the vacuum state of the field can depend on the choice of the inertial reference frame.

\section{ACKNOWLEDGMENTS}

This work was supported by the Air Force Office of Scientific Research (Award No. FA9550-18-1-0141), the Office of Naval Research (Awards No. N00014-16-1-3054 and No. N00014-16-1-2578), the Robert A. Welch Foundation (Award No. A-1261), and Fujian 100-talents program.
[1] W. G. Unruh, Notes on black hole evaporation, Phys. Rev. D 14, 870 (1976).

[2] S. A. Fulling, Nonuniqueness of canonical field quantization in Riemannian space-time, Phys. Rev. D 7, 2850 (1973).

[3] P. C. W. Davies, Scalar production in Schwarzschild and Rindler metrics, J. Phys. A 8, 609 (1975).

[4] W. Rindler, Kruskal space and the uniformly accelerated frame, Am. J. Phys. 34, 1174 (1966).

[5] W. G. Unruh and R. M. Wald, What happens when an accelerating observer detects a Rindler particle, Phys. Rev. D 29, 1047 (1984).

[6] M. O. Scully, S. Fulling, D. M. Lee, D. N. Page, W. P. Schleich, and A. A. Svidzinsky, Quantum optics approach to radiation from atoms falling into a black hole, Proc. Natl. Acad. Sci. USA 115, 8131 (2018).

[7] A. A. Svidzinsky, J. S. Ben-Benjamin, S. A. Fulling, and D. N. Page, Excitation of an Atom by a Uniformly Accelerated Mirror through Virtual Transitions, Phys. Rev. Lett. 121, 071301 (2018).

[8] A. A. Sokolov and I. M. Ternov, On polarization and spin effects in the theory of synchrotron radiation, Sov. Phys. Dokl. 8, 1203 (1964).

[9] J. S. Bell and J. M. Leinaas, Electrons as accelerated thermometers, Nucl. Phys. B 212, 131 (1983).

[10] J. S. Bell and J. M. Leinaas, The Unruh effect and quantum fluctuations of electrons in storage rings, Nucl. Phys. B 284, 488 (1987)

[11] W. G. Unruh, Acceleration radiation for orbiting electrons, Phys. Rep. 307, 163 (1998).

[12] E. T. Akhmedov and D. Singleton, On the relation between Unruh and Sokolov-Ternov effects, Int. J. Mod. Phys. A 22, 4797 (2007).

[13] M. O. Scully, V. V. Kocharovsky, A. Belyanin, E. Fry, and F. Capasso, Enhancing Acceleration Radiation from GroundState Atoms via Cavity Quantum Electrodynamics, Phys. Rev. Lett. 91, 243004 (2003); A. Belyanin, V. V. Kocharovsky, F.
Capasso, E. Fry, M. S. Zubairy, and M. O. Scully, Quantum electrodynamics of accelerated atoms in free space and in cavities, Phys. Rev. A 74, 023807 (2006).

[14] V. L. Ginzburg and V. P. Frolov, Excitation and emission of a "detector" in accelerated motion in a vacuum or in uniform motion at a velocity above the velocity of light in a medium, Pis'ma Zh. Eksp. Teor. Fiz. 43, 265 (1986) [JETP Lett. 43, 339 (1986)].

[15] V. P. Frolov and V. L. Ginzburg, Excitation and radiation of an accelerated detector and anomalous Doppler effect, Phys. Lett. A 116, 423 (1986)

[16] I. Brevik and H. Kolbenstvedt, Quantum point detector moving through a dielectric medium, Nuovo Cimento B 102, 139 (1988).

[17] J. M. Jauch and K. M. Watson, Phenomenological quantum electrodynamics, Phys. Rev. 74, 950 (1948).

[18] T. Musha, Unified consideration of wave amplification in moving media, J. Appl. Phys. 35, 3273 (1964).

[19] A. H. W. Beck, Space-Charge Waves and Slow Electromagnetic Waves (Pergamon Press, Inc., New York, 1958).

[20] C. K. Birdsall, G. R. Brewer, and A. V. Haeff, The resistive-wall amplifier, Proc. IRE 41, 865 (1953).

[21] A. R. Hutson, J. H. McFee, and D. L. White, Ultrasonic Amplification in CdS, Phys. Rev. Lett. 7, 237 (1961).

[22] J. B. Pendry, Shearing the vacuum: Quantum friction, J. Phys.: Condens. Matter 9, 10301 (1997).

[23] A. I. Volokitin and B. N. J. Persson, Near-field radiative heat transfer and noncontact friction, Rev. Mod. Phys. 79, 1291 (2007).

[24] S. A. R. Horsley, Canonical quantization of the electromagnetic field interacting with a moving dielectric medium, Phys. Rev. A 86, 023830 (2012).

[25] Y. Guo and Z. Jacob, Singular evanescent wave resonances in moving media, Opt. Express 22, 26193 (2014).

[26] A. Manjavacas and F. J. Garcia de Abajo, Vacuum Friction in Rotating Particles, Phys. Rev. Lett. 105, 113601 (2010). 
[27] M. F. Maghrebi, R. L. Jaffe, and M. Kardar, Spontaneous Emission by Rotating Objects: A Scattering Approach, Phys. Rev. Lett. 108, 230403 (2012).

[28] R. Zhao, A. Manjavacas, F. J. Garcia de Abajo, and J. B. Pendry, Rotational Quantum Friction, Phys. Rev. Lett. 109, 123604 (2012).

[29] S. W. Hawking, Black hole explosions? Nature (London) 248, 30 (1974).

[30] C. Barcelo, S. Liberati, and M. Visser, Analogue gravity, Living Rev. Relativ. 14, 3 (2011).

[31] T. G. Philbin, C. Kuklewicz, S. Robertson, S. Hill, F. Konig, and U. Leonhardt, Fiber-optical analog of the event horizon, Science 319, 1367 (2008).

[32] T. Dodo, Quantum theory of negative energy in microwave tubes, Jpn. J. Appl. Phys. 3, 16 (1964).

[33] C. Luo, M. Ibanescu, S. G. Johnson, and J. D. Joannopoulos, Cherenkov radiation in photonic crystals, Science 299, 368 (2003).

[34] J. M. Pitarke, V. M. Silkin, E. V. Chulkov, and P. M. Echenique, Theory of surface plasmons and surface-plasmon polaritons, Rep. Prog. Phys. 70, 1 (2007).
[35] M. G. Silveirinha, Optical Instabilities and Spontaneous Light Emission by Polarizable Moving Matter, Phys. Rev. X 4, 031013 (2014).

[36] S. Lannebère and M. G. Silveirinha, Negative spontaneous emission by a moving two-level atom, J. Opt. 19, 014004 (2017).

[37] M. G. Silveirinha, Spontaneous parity-time-symmetry breaking in moving media, Phys. Rev. A 90, 013842 (2014).

[38] M. Wallquist, K. Hammerer, P. Rabl, M. Lukin, and P. Zoller, Hybrid quantum devices and quantum engineering, Phys. Scr. T137, 014001 (2009).

[39] H. B. G. Casimir and D. Polder, The influence of retardation on the London-van der Waals forces, Phys. Rev. 73, 360 (1948).

[40] For the density matrix formulation of the quantum theory of the laser, see M. Scully and W. Lamb Jr., Quantum Theory of an Optical Maser, Phys. Rev. Lett. 16, 853 (1966). For pedagogical treatment and references, see E. R. Pike and S. Sarkar, The Quantum Theory of Radiation (Oxford University Press, New York, 1996); M. Scully and S. Zubairy, Quantum Optics (Cambridge University Press, Cambridge, 1997). 